\title{
Improvement of weighted compact scheme with multi-step strategy for supersonic compressible flow
}

\author{
Jun Peng, Yiqing Shen* \\ State Key Laboratory of High Temperature Gas Dynamics, Institute of Mechanics, Chinese Academy of Sciences, Beijing 100190, China
}

\section{A R T I C L E I N F O}

\section{Article history:}

Received 14 July 2014

Received in revised form 8 April 2015

Accepted 11 April 2015

Available online 18 April 2015

\section{Keywords:}

Numerical method

Supersonic compressible flow

Weighted compact scheme

Multi-step strategy

\begin{abstract}
A B S T R A C T
With shock capturing capability and spectral like resolution, weighted compact schemes are good choices for supersonic turbulent flow simulation. However, because of the implicit nature of compact schemes, accuracy decrease at one point would contaminate the overall solution. The recently proposed compact-reconstructed WENO (CRWENO) scheme (Ghosh and Baeder, 2012) is an efficient weighted compact scheme. Analysis shows that the accuracy of the CRWENO scheme is only second order for points near discontinuities. In this paper, a multi-step strategy is proposed to increase the accuracy of the CRWENO scheme near discontinuities. Instead of constructing the fifth order compact scheme directly from three third order compact schemes, two fourth order compact schemes are first constructed. The final fifth order scheme is then obtained by these two fourth order schemes. In this way, the accuracy of the CRWENO scheme is increased to third order for points near discontinuities. The dissipation and dispersion properties of the new scheme are analyzed by employing the modified wave number method. Several examples, including linear advection cases, three one-dimensional shock tube problems, twodimensional isentropic vortex convection, double Mach reflection, and shock/mixing layer interaction problems, are considered to assess the present method. Numerical results show that the present multi-step CRWENO scheme has lower dissipation and higher resolution in both smooth and discontinuous regions compared with the original scheme.
\end{abstract}

() 2015 Elsevier Ltd. All rights reserved.

\section{Introduction}

The numerical simulation of supersonic turbulence is still a challenging field for both scientists and engineers. As shock and multi-scale problems present simultaneously in supersonic turbulent flow, numerical schemes are required to be able to resolve both of these two phenomena at the same time. Generally, for multi-scale problems such as turbulence and acoustics, minimal dissipation and dispersion are demanded to ensure high resolution of the wave number range that considered. For discontinuities such as shock waves, a numerical method should remove spurious numerical oscillation by introducing numerical dissipation which leads to high dissipation near discontinuity as well as in smooth regions of the flow field. Due to the contradictory requirements of these two phenomena for numerical methods, most existing methods may only be successful in handling one of the problems.

The purpose of developing shock capturing schemes is to resolve discontinuities without numerical oscillation while keeping high accuracy. The most influential shock capturing scheme

\footnotetext{
* Corresponding author.

E-mail addresses: pengjun@imech.ac.cn (J. Peng), yqshen@imech.ac.cn (Y. Shen).
}

at present is the weighted essentially non-oscillatory scheme introduced by Jiang and Shu [1] (referred as WENO-JS). Based on the ENO (Essentially non-oscillatory) scheme by Harten et al. [2] and the finite volume WENO scheme by Liu et al. [3], it is developed in the frame of finite difference and more efficient for multi-dimensional problems. With improved smoothness indicators, the WENO-JS scheme captures discontinuity well and maintains high order in smooth region compared to traditional low order schemes such as TVD schemes [4] and ENO schemes [2]. In spite of these advantages, numerical experiments show that the smoothness indicator of WENO-JS is too dissipative for complex flow structures such as turbulence [5]. Moreover, the weighting function of WENO-JS fails to recover designed coefficients at critical points where the first derivative equals 0 . To improve the accuracy of WENO-JS at critical points, Henrick et al. [6] proposed a mapping function for the original weights of WENO-JS. However, the additional computational cost brought by the mapping function is not preferable. Another approach is the WENO-Z scheme of Borges et al. [7] which employs a new weighting function and achieves low dissipation and ideal order at low computational cost. Yamaleev and Carpenter [8] proposed the ESWENO scheme which guarantees designed order for arbitrary number of zero derivatives. 
Martin et al. [9] developed a set of symmetric WENO schemes for direct and large eddy simulation of compressive turbulence. Martin's schemes are optimized to achieve maximum order of accuracy and bandwidth with minimized dissipation. Nevertheless, Shen and Zha [10] showed that the accuracy of above mentioned WENO schemes is only second order near discontinuities.

For multi-scale dominated problems, the compact scheme is a better choice than the shock capturing scheme in terms with wave resolution. The compact scheme did not draw much attention until Lele's pioneering work [11]. He developed a class of central compact schemes with spectral-like resolution. Based on Fourier analysis, Lele also analyzed the spectral behavior of different approximations. After that compact schemes were widely used in turbulence [12,13] and acoustics simulations [14]. Inspired by Lele's central compact scheme, different kinds of compact schemes $[12,15,16]$ were developed with optimized parameters to minimize dissipation and dispersion. Tam and Webb [17] showed that even the optimized compact scheme of Lele may produce incorrect wave speed, and the dispersion-relation-preserving (DRP) finite-difference scheme was proposed to solve this problem and improve resolution. For hyperbolic conservation laws such as the Euler equations, upwind compact schemes were developed [18-21] and it was shown that they are more robust than the central compact schemes for advection dominated problems. Although compact schemes have high resolution for multi scale problems, they incur oscillations near shock and thus are not suitable for supersonic flow simulation.

In order to capture shock wave without numerical oscillation while taking advantage of the spectral-like resolution of compact schemes, a class of hybrid schemes is proposed by combining the shock capturing scheme with the compact scheme. Adams and Shariff [22] developed a hybrid compact-ENO scheme coupled by a simple detection method for shock turbulence interaction simulation. Pirozzoli [23] followed Adams's method and proposed a compact-WENO scheme with a similar criterion. Ren et al. [24] introduced a more sophisticated switch function and developed a characteristic-wise hybrid compact-WENO scheme. Hill and Pullin [25] coupled the tuned center-difference scheme with the WENO scheme that a switch indicator based on the smoothness indicator of WENO was used. Kim and Kwon [26] combined several central difference schemes with the WENO scheme using the same switch function as Ren et al. All above mentioned switch functions always need an artificial (or empirical), problem-dependent parameter to determine whether the shock capturing scheme should be used. This may cause some problems such as loss of accuracy and robustness and is inefficient in parallel computation [27]. To develop parameter free hybrid schemes, a function based on the WENO smoothness indicators of Jiang and Shu [1] and Borges et al. [7] was proposed to detect shock regions by Shen and Zha [28], and a high resolution finite compact scheme was constructed which treats detected discontinuity as internal boundary.

A way to avoid switch functions is to construct a compact scheme based on the idea of WENO schemes and ENO schemes. Jiang et al. [29] developed a weighted compact scheme based on Lele's central compact scheme. This scheme is a weighted combination of several lower order compact schemes on different substencils. Deng and Zhang [30] developed a weighted compact scheme that the cell edge flux of a cell centered compact scheme is approximated by the WENO scheme. Zhang et al. [27] extended Deng's scheme to higher orders. Recently, a more efficient fifth order compact reconstructed WENO scheme (CRWENO) was proposed by Ghosh and Baeder [31]. The reconstruction process of this scheme is similar to that of Jiang's but in conservative form that the final fifth order compact scheme is constructed by three third order compact schemes. The CRWENO scheme has good shock capturing capability and high wave resolution. Numerical experiments [32] showed that the CRWENO scheme is capable of resolving finer wave structures than the WENO scheme at the same grid size.

As analyzed by Shen and Zha [10], a $2 r+1$ th order WENO scheme which is constructed directly from $r$ candidate fluxes may decrease the accuracy near discontinuities, so does the CRWENO scheme. For the traditional WENO scheme, the reconstructed flux at the cell interface is an explicit interpolation of cell central flux values, accuracy decrease at one point has few effect on the accuracy of other points during the reconstruction process. However, due to the implicit feature of the compact scheme, accuracy decrease at one point would contaminate all points. For direct and large eddy simulation of supersonic turbulence, shocklets $[33,34,13]$ are fundamental and important phenomena. Numbers of shocklets may simultaneously appear in supersonic turbulent flow. Serious accuracy decrease due to shocklets may invalidate the numerical simulation if one applies the weighted compact scheme for such flow. Therefore, the improvement of the accuracy near discontinuities is vital. In this paper, we analyze the accuracy of the CRWENO scheme near discontinuities, and then a multi-step method is introduced to improve the accuracy of the CRWENO scheme. Several numerical examples are presented to show that the new scheme is efficient and accurate.

This paper is organized as follows. Section 2 describes the construction process of the CRWENO scheme. Section 3 analyzes the accuracy of the CRWENO scheme near discontinuities. In Section 4, a multi-step CRWENO scheme is constructed to improve the original scheme. Numerical results of scalar equation, onedimensional and two-dimensional Euler equations as well as twodimensional Navier-Stokes equations are showed in Section 5. Finally, discussion and conclusion are presented in Section 6.

\section{The CRWENO scheme of Ghosh and Baeder}

To describe the CRWENO scheme, we consider a general scalar conservation law in $1 \mathrm{D}$ expressed as:

$u_{t}+f_{x}=0$

where $u(x, t)$ is the conserved variable and $f(u)$ is the flux function. To solve (1) numerically, we transform it into semi-discretized form for uniformly discretized space:

$\frac{d u_{i}}{d t}=-\frac{\hat{f}_{i+\frac{1}{2}}-\hat{f}_{i-\frac{1}{2}}}{\Delta x}$

in which $u_{i}$ is the numerical approximation of $u$ at $x_{i}, \hat{f}_{i+\frac{1}{2}}$ is the numerical flux of $f$ at cell interface $x_{i+\frac{1}{2}}$ and $\Delta x=x_{i+1}-x_{i}$. The numerical flux $\hat{f}_{i+\frac{1}{2}}$ at cell interface is interpolated with discrete $f_{i}$ values at $x_{i}$. The linear fifth order compact scheme is expressed as:

$\frac{3}{10} \hat{f}_{i-\frac{1}{2}}+\frac{6}{10} \hat{f}_{i+\frac{1}{2}}+\frac{1}{10} \hat{f}_{i+\frac{3}{2}}=\frac{1}{30} f_{i-1}+\frac{19}{30} f_{i}+\frac{10}{30} f_{i+1}$

(3) can be obtained from the weighted sum of three third order compact schemes:

$\frac{2}{3} \hat{f}_{i-\frac{1}{2}}+\frac{1}{3} \hat{f}_{i+\frac{1}{2}}=\frac{1}{6} f_{i-1}+\frac{5}{6} f_{i}$

$\frac{1}{3} \hat{f}_{i-\frac{1}{2}}+\frac{2}{3} \hat{f}_{i+\frac{1}{2}}=\frac{5}{6} f_{i}+\frac{1}{6} f_{i+1}$

$\frac{2}{3} \hat{f}_{i+\frac{1}{2}}+\frac{1}{3} \hat{f}_{i+\frac{3}{2}}=\frac{1}{6} f_{i}+\frac{5}{6} f_{i+1}$

with weights $c_{0}=\frac{2}{10}, c_{1}=\frac{5}{10}$ and $c_{2}=\frac{3}{10}$ respectively. 
Based on the idea of the WENO scheme, the CRWENO scheme [31] is constructed as:

$$
\begin{gathered}
\left(\frac{2}{3} \omega_{0}+\frac{1}{3} \omega_{1}\right) \hat{f}_{i-\frac{1}{2}}+\left[\frac{1}{3} \omega_{0}+\frac{2}{3}\left(\omega_{1}+\omega_{2}\right)\right] \hat{f}_{i+\frac{1}{2}}+\frac{1}{3} \omega_{3} \hat{f}_{i+\frac{3}{2}} \\
=\frac{\omega_{0}}{6} f_{i-1}+\frac{5\left(\omega_{0}+\omega_{1}\right)+\omega_{2}}{6} f_{i-1}+\frac{\omega_{1}+5 \omega_{2}}{6} f_{i-1}
\end{gathered}
$$

where the weights $\omega_{k}$ are defined as:

$\omega_{k}=\frac{a_{k}}{\sum_{i} a_{i}}$

$a_{k}=\frac{c_{k}}{\left(\varepsilon+\beta_{k}\right)^{p}}$

in which

$\beta_{0}=\frac{13}{12}\left(f_{i-2}-2 f_{i-1}+f_{i}\right)^{2}+\frac{1}{4}\left(f_{i-2}-4 f_{i-1}+3 f_{i}\right)^{2}$

$\beta_{1}=\frac{13}{12}\left(f_{i-1}-2 f_{i}+f_{i+1}\right)^{2}+\frac{1}{4}\left(f_{i-1}-f_{i+1}\right)^{2}$

$\beta_{2}=\frac{13}{12}\left(f_{i}-2 f_{i+1}+f_{i+2}\right)^{2}+\frac{1}{4}\left(3 f_{i}-4 f_{i+1}+f_{i+2}\right)^{2}$

where $\beta_{k}$ are the smoothness indicators [1] and $\varepsilon$ is a small number to avoid division by zero.

For common construction procedure of the WENO scheme [1], it has been shown that the weighting function (7) is too dissipative and does not guarantee fifth order accuracy when the first derivative approximates 0. A mapping function [6] is introduced to improve the accuracy of the final weights:

$g_{k}(\omega)=\frac{\omega\left(c_{k}+c_{k}^{2}-3 c_{k} \omega+\omega_{2}\right)}{c_{k}^{2}+\omega\left(1-2 c_{k}\right)}$

$\omega_{k}^{M}=\frac{g_{k}}{\sum_{i} g_{i}}$

However, mapping function (9) leads to much computational expense. An alternative weight [7] is given by:

$a_{k}=c_{k}\left(1+\frac{\tau}{\beta_{k}+\varepsilon}\right)$

where $\tau=\left|\beta_{0}-\beta_{2}\right|$. Another important issue about the implementation of the WENO weights is the choice of the small number $\varepsilon$. For the weights of Jiang and Shu, $\varepsilon=1 \times 10^{-6}$ is advised. For weight (9) and (11), $1 \times 10^{-40}$ or $1 \times 10^{-20}$ (depending on the structure of computers) is recommended. In this paper, $\varepsilon$ is set to be $1 \times 10^{-6}$ for all numerical experiments. Readers may refer to [10] for discussion on the weighting function and $\varepsilon$.

\section{Accuracy analysis at points near discontinuities}

Compared to the fifth order WENO scheme, the CRWENO scheme has better resolution and dissipation properties [31]. However, as shown below, the accuracy near discontinuities is not ideal. Due to the implicit feature of compact schemes, accuracy decrease at one point would decrease the overall accuracy. This is a main drawback of the CRWENO scheme as well as for other weighted compact schemes. For complex flow such as shock/ boundary layer interaction and supersonic turbulent flow with shocklets, the flow field we are interested in always lies near or between discontinuities.

For the 5th order CRWENO scheme, there are five points involved to calculate the numerical flux. Here, as an example, we evaluate the accuracy at such a model stencil that there are 6 points between two discontinuities (see Fig. 1). For each of the two points at the left and right boundaries (point 0 and 5), only one 3-point candidate stencil is smooth, i.e., $\left(x_{0}, x_{1}, x_{2}\right)$ and $\left(x_{3}, x_{4}, x_{5}\right)$. For point 0 , discontinuity lies in its first and second candidate stencils thus $\omega_{0} \rightarrow 0, \omega_{1} \rightarrow 0$ and $\omega_{2} \rightarrow 1$. Therefore, at point 0 , (3) becomes:

$\frac{2}{3} \hat{f}_{0+\frac{1}{2}}+\frac{1}{3} \hat{f}_{0+\frac{3}{2}}=\frac{1}{6} f_{0}+\frac{5}{6} f_{1}$

For point 1 , discontinuity lies in its first stencil. Then we have $\omega_{0} \rightarrow 0, \omega_{1} \rightarrow \frac{5}{8}, \omega_{2} \rightarrow \frac{3}{8}$ and

$\frac{5}{24} \hat{f}_{1-\frac{1}{2}}+\frac{2}{3} \hat{f}_{1+\frac{1}{2}}+\frac{3}{24} \hat{f}_{1+\frac{3}{2}}=\frac{28}{48} f_{1}+\frac{20}{48} f_{2}$

Following the above process for remainder of the points we obtain:

$\frac{3}{10} \hat{f}_{2-\frac{1}{2}}+\frac{6}{10} \hat{f}_{2+\frac{1}{2}}+\frac{3}{24} \hat{f}_{2+\frac{3}{2}}=\frac{1}{30} f_{1}+\frac{19}{30} f_{2}+\frac{1}{3} f_{3}$

$\frac{3}{10} \hat{f}_{3-\frac{1}{2}}+\frac{6}{10} \hat{f}_{3+\frac{1}{2}}+\frac{3}{24} \hat{f}_{3+\frac{3}{2}}=\frac{1}{30} f_{2}+\frac{19}{30} f_{3}+\frac{1}{3} f_{4}$

$\frac{9}{21} \hat{f}_{4-\frac{1}{2}}+\frac{12}{21} \hat{f}_{4+\frac{1}{2}}=\frac{2}{42} f_{3}+\frac{35}{42} f_{4}+\frac{5}{42} f_{5}$

$\frac{2}{3} \hat{f}_{5-\frac{1}{2}}+\frac{1}{3} \hat{f}_{5+\frac{1}{2}}=\frac{1}{6} f_{4}+\frac{5}{6} f_{5}$

(12)-(17) can be written in matrix form:

$$
\begin{aligned}
& \left(\begin{array}{cccccc}
2 / 3 & 1 / 3 & 0 & 0 & 0 & 0 \\
5 / 24 & 2 / 3 & 3 / 24 & 0 & 0 & 0 \\
0 & 3 / 10 & 6 / 10 & 1 / 10 & 0 & 0 \\
0 & 0 & 3 / 10 & 6 / 10 & 1 / 10 & 0 \\
0 & 0 & 0 & 9 / 21 & 12 / 21 & 0 \\
0 & 0 & 0 & 0 & 2 / 3 & 1 / 3
\end{array}\right)\left(\begin{array}{l}
\hat{f}_{0+\frac{1}{2}} \\
\hat{f}_{1+\frac{1}{2}} \\
\hat{f}_{2+\frac{1}{2}} \\
\hat{f}_{3+\frac{1}{2}} \\
\hat{f}_{4+\frac{1}{2}} \\
\hat{f}_{5+\frac{1}{2}}
\end{array}\right) \\
& =\left(\begin{array}{cccccc}
1 / 6 & 5 / 6 & 0 & 0 & 0 & 0 \\
0 & 28 / 48 & 20 / 48 & 0 & 0 & 0 \\
0 & 1 / 30 & 19 / 30 & 1 / 3 & 0 & 0 \\
0 & 0 & 1 / 30 & 19 / 30 & 1 / 3 & 0 \\
0 & 0 & 0 & 2 / 42 & 35 / 42 & 5 / 42 \\
0 & 0 & 0 & 0 & 1 / 6 & 5 / 6
\end{array}\right)\left(\begin{array}{l}
f_{0} \\
f_{1} \\
f_{2} \\
f_{3} \\
f_{4} \\
f_{5}
\end{array}\right)
\end{aligned}
$$

Note that $\hat{f}_{i+1 / 2}-\hat{f}_{i-1 / 2}=\Delta x f_{i}^{\prime}$, we define a matrix $C$ :

$C=\left[\begin{array}{cccccc}0 & 0 & 0 & 0 & 0 & 0 \\ -1 & 1 & & & & \\ & -1 & 1 & & & \\ & & -1 & 1 & & \\ & & & -1 & 1 & \\ & & & & -1 & 1\end{array}\right]$

Left-multiplying matrix $C$ to each side of (18), we obtain the linear equations about $f_{i}^{\prime}$ :

$\left[\begin{array}{l}0 \\ f_{1}^{\prime} \\ f_{2}^{\prime} \\ f_{3}^{\prime} \\ f_{4}^{\prime} \\ f_{5}^{\prime}\end{array}\right]=\frac{1}{\Delta x}\left[\begin{array}{cccccc}0 & 0 & 0 & 0 & 0 & 0 \\ -1 & 1 & & & & \\ & -1 & 1 & & & \\ & & -1 & 1 & & \\ & & & -1 & 1 & \\ & & & & -1 & 1\end{array}\right]\left[\begin{array}{l}\hat{f}_{0+\frac{1}{2}} \\ \hat{f}_{1+\frac{1}{2}} \\ \hat{f}_{2+\frac{1}{2}} \\ \hat{f}_{3+\frac{1}{2}} \\ \hat{f}_{4+\frac{1}{2}} \\ \hat{f}_{5+\frac{1}{2}}\end{array}\right]=\frac{1}{\Delta x} C A^{-1} B\left[\begin{array}{l}f_{0} \\ f_{1} \\ f_{2} \\ f_{3} \\ f_{4} \\ f_{5}\end{array}\right]$ 


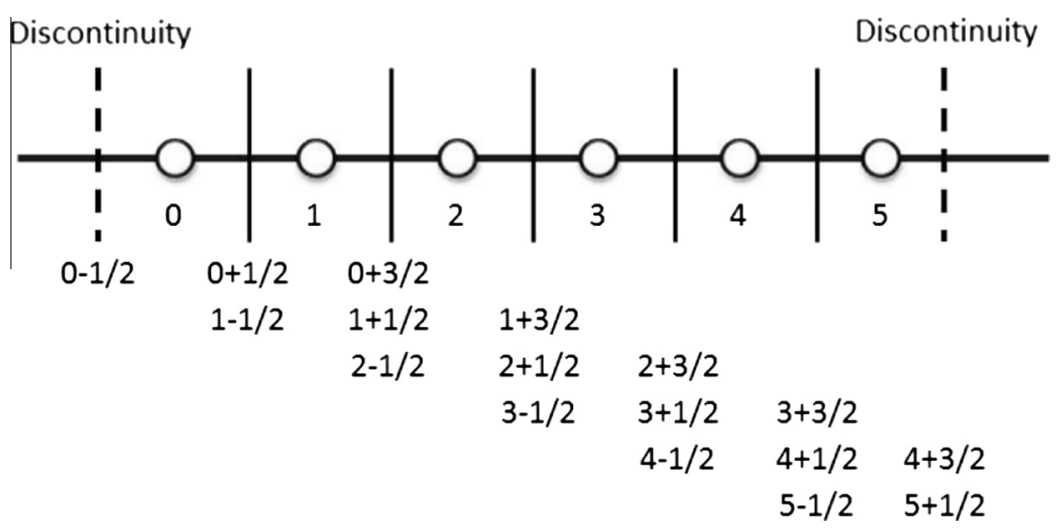

Fig. 1. Sketch of six points between two discontinuities.

where

$C A^{-1} B=\left(\begin{array}{cccccc}0 & 0 & 0 & 0 & 0 & 0 \\ -49 / 120 & -7 / 24 & 149 / 180 & -3 / 20 & 1 / 40 & -1 / 360 \\ 59 / 360 & -67 / 72 & 54 / 161 & 11 / 20 & -11 / 120 & -11 / 1080 \\ -11 / 120 & 11 / 20 & -229 / 180 & 29 / 60 & 19 / 40 & -19 / 360 \\ 7 / 120 & -7 / 24 & 133 / 180 & -31 / 20 & 91 / 120 & 103 / 360 \\ -3 / 40 & 3 / 8 & -19 / 20 & 37 / 20 & -199 / 40 & 71 / 40\end{array}\right)$

Therefore, the first derivatives of numerical fluxes $f_{i}$ are now represented by values at each point. To evaluate the accuracy of $f_{i}^{\prime}$, we employ Taylor expansion to each $f_{i}^{\prime}$ obtained by (20) and yield:

$f_{1}^{\prime}=\left.\frac{\partial f}{\partial x}\right|_{1}+\frac{4}{45} f_{1}^{(3)} \Delta x^{2}+O\left(\Delta x^{3}\right)$

$f_{2}^{\prime}=\left.\frac{\partial f}{\partial x}\right|_{2}-\frac{13}{270} f_{2}^{(3)} \Delta x^{2}+O\left(\Delta x^{3}\right)$

$f_{3}^{\prime}=\left.\frac{\partial f}{\partial x}\right|_{3}+\frac{1}{45} f_{3}^{(3)} \Delta x^{2}+O\left(\Delta x^{3}\right)$

$f_{4}^{\prime}=\left.\frac{\partial f}{\partial x}\right|_{4}+\frac{1}{90} f_{4}^{(3)} \Delta x^{2}+O\left(\Delta x^{3}\right)$

$f_{5}^{\prime}=\left.\frac{\partial f}{\partial x}\right|_{5}-\frac{2}{15} f_{5}^{(3)} \Delta x^{2}+O\left(\Delta x^{3}\right)$

(22a)-(22e), show that at points $1-5$ the accuracy is only second order. As the relation between neighboring points is determined by (20), when one of its neighboring points has a low accuracy order, the accuracy of a certain point is also decreased, even for smooth region like point 3.

\section{Multi-step weighting method for CRWENO}

As mentioned above, the accuracy of points near discontinuities is only second order. To improve that, a multi-step reconstruction process is proposed. Firstly, two fourth order schemes are constructed by using two of the three candidate third order schemes. For example, a fourth order flux (25) is constructed from (23) and (24), i.e., $(25)=(23) \times c_{0}^{0}+(24) \times c_{1}^{0}$. Note here, without loss of accuracy and spectral-like resolution, (4b) is replaced with (24) or (26) during the construction process based on upwinding consideration.

$\frac{2}{3} \hat{f}_{i-\frac{1}{2}}+\frac{1}{3} \hat{f}_{i+\frac{1}{2}}=\frac{1}{6} f_{i-1}+\frac{5}{6} f_{i}, \quad c_{0}^{0}=\frac{3}{4}$ $\hat{f}_{i+\frac{1}{2}}=\frac{2}{6} f_{i+1}+\frac{5}{6} f_{i}-\frac{1}{6} f_{i-1}, \quad c_{1}^{0}=\frac{1}{4}$

$\frac{1}{2} \hat{f}_{i-\frac{1}{2}}+\frac{1}{2} \hat{f}_{i+\frac{1}{2}}=\frac{1}{12} f_{i+1}+\frac{5}{6} f_{i}+\frac{1}{12} f_{i-1}$

Similarly, for the second and third stencils we have:

$\hat{f}_{i+\frac{1}{2}}=\frac{2}{6} f_{i+1}+\frac{5}{6} f_{i}-\frac{1}{6} f_{i-1}, \quad c_{0}^{1}=\frac{1}{4}$

$\frac{2}{3} \hat{f}_{i+\frac{1}{2}}+\frac{1}{3} \hat{f}_{i+\frac{3}{2}}=\frac{5}{6} f_{i+1}+\frac{1}{6} f_{i}, \quad c_{1}^{1}=\frac{3}{4}$

$\frac{3}{4} \hat{f}_{i+\frac{1}{2}}+\frac{1}{4} \hat{f}_{i+\frac{3}{2}}=\frac{17}{24} f_{i+1}+\frac{8}{24} f_{i}-\frac{1}{24} f_{i-1}$

Then these two fourth order schemes, (25) and (28), are used to construct the final fifth order scheme with coefficients $c_{0}$ and $c_{1}$ :

$\frac{1}{2} \hat{f}_{i-\frac{1}{2}}+\frac{1}{2} \hat{f}_{i+\frac{1}{2}}=\frac{1}{12} f_{i+1}+\frac{5}{6} f_{i}+\frac{1}{12} f_{i-1}, \quad c_{0}=0.6$
$\frac{3}{4} \hat{f}_{i+\frac{1}{2}}+\frac{1}{4} \hat{f}_{i+\frac{3}{2}}=\frac{17}{24} f_{i+1}+\frac{8}{24} f_{i}-\frac{1}{24} f_{i-1}, \quad c_{1}=0.4$
$\frac{3}{10} \hat{f}_{i+\frac{1}{1}}+\frac{6}{10} \hat{f}_{i+\frac{1}{1}}+\frac{1}{10} \hat{f}_{i+\frac{1}{1}}=\frac{1}{30} f_{i-1}+\frac{19}{30} f_{i}+\frac{10}{30} f_{i+1}$

That is $(29)=(25) \times c_{0}+(28) \times c_{1}$. Replacing the optimal weights with the non-linear weighting functions in (25), (28) and (29), we have the final fifth order weighted compact scheme

$a_{i} \hat{f}_{i-\frac{1}{2}}+b_{i} \hat{f}_{i+\frac{1}{2}}+c_{i} \hat{f}_{i+\frac{3}{2}}=d_{i+\frac{1}{2}}$

where

$a_{i}=\frac{2}{3} \omega_{0} \omega_{0}^{0}$

$b_{i}=\frac{1}{3}\left(\omega_{0} \omega_{0}^{0}+3 \omega_{0} \omega_{1}^{0}+3 \omega_{1} \omega_{0}^{1}+2 \omega_{1} \omega_{1}^{1}\right)$

$c_{i}=\frac{1}{3} \omega_{1} \omega_{1}^{1}$

$$
\begin{aligned}
d_{i+\frac{1}{2}}= & \frac{1}{6}\left(\omega_{0} \omega_{0}^{0}-\omega_{0} \omega_{1}^{0}-\omega_{1} \omega_{0}^{1}\right) f_{i-1}+\frac{1}{6}\left(5 \omega_{0} \omega_{0}^{0}+5 \omega_{0} \omega_{1}^{0}\right. \\
& \left.+5 \omega_{1} \omega_{0}^{1}+\omega_{1} \omega_{1}^{1}\right) f_{i}+\frac{1}{6}\left(2 \omega_{0} \omega_{1}^{0}+2 \omega_{1} \omega_{1}^{1}\right. \\
& \left.+5 \omega_{1} \omega_{1}^{1}\right) f_{i+1}
\end{aligned}
$$

To ensure the convergence condition:

$\omega_{k}-c_{k}=O\left(\Delta x^{3}\right)$ 
the mapping function (9) is employed at each step when computing the nonlinear weights. The non-linear weights are redefined as:

$$
\begin{aligned}
& \omega_{k}^{l}=\frac{g\left(\psi_{k}^{l}\right)}{\sum_{i} g\left(\psi_{i}^{l}\right)}, \psi_{k}^{l}=\frac{a_{k}^{l}}{\sum_{i} a_{i}^{l}}, \quad a_{k}^{l}=c_{k}^{l}\left(1+\frac{\tau_{4}^{l}}{\beta_{k+l}+\varepsilon}\right), \\
& \tau_{4}^{l}=\left|\beta_{l+1}-\beta_{l}\right|, \quad k=0,1, \quad l=0,1 \\
& \omega_{k}=\frac{g\left(\psi_{m}\right)}{\sum_{i} g\left(\psi_{i}\right)}, \psi_{m}=\frac{a_{m}}{\sum_{i} a_{i}}, \quad a_{m}=c_{m}\left(1+\frac{\tau_{5}}{\beta_{2 m}+\varepsilon}\right), \\
& \tau_{5}=\left|\beta_{2}-\beta_{0}\right|, m=0,1
\end{aligned}
$$

Definitions of $\beta_{k}$ are the same as $(8 \mathrm{a})-(8 \mathrm{c})$.

Following the same process in Section 3, the accuracy of the present scheme can be evaluated. Here we omit the deduction process and present the result directly. At each point defined in Fig. 1 there are:

$f_{1}^{\prime}=\left.\frac{\partial f}{\partial x}\right|_{1}+\frac{1}{24} f_{1}^{(3)} \Delta x^{2}+O\left(\Delta x^{3}\right)$

$f_{2}^{\prime}=\left.\frac{\partial f}{\partial x}\right|_{2}+\frac{13}{396} f_{2}^{(4)} \Delta x^{3}+O\left(\Delta x^{4}\right)$

$f_{3}^{\prime}=\left.\frac{\partial f}{\partial x}\right|_{3}-\frac{1}{44} f_{3}^{(4)} \Delta x^{3}+O\left(\Delta x^{4}\right)$

$f_{4}^{\prime}=\left.\frac{\partial f}{\partial x}\right|_{4}+\frac{5}{132} f_{4}^{(4)} \Delta x^{3}+O\left(\Delta x^{4}\right)$

$f_{5}^{\prime}=\left.\frac{\partial f}{\partial x}\right|_{5}-\frac{1}{12} f_{5}^{(3)} \Delta x^{2}+O\left(\Delta x^{3}\right)$

(36a)-(36e), show that the present method improves the accuracy of $f_{2}^{\prime}, f_{3}^{\prime}$ and $f_{4}^{\prime}$ from second order to third order. Besides, the magnitudes of the leading error terms are also halved in (36a) and (36e) compared to (22a) and (22e).

The dispersion and dissipation properties of a certain scheme can be obtained by applying Fourier analysis [11]. Consider the Fourier decomposition of a periodic flux function $f_{N}(x)$ on a uniformly spaced grid:

$f_{N}(x)=\sum_{k=0}^{N-1} \hat{f}_{k} \exp \left(\frac{2 \pi i k x}{L}\right)$

where $i=\sqrt{-1}$. One obtains the Fourier decomposition of the first derivative of $f_{N}(x)$ by differentiating (37):

$f_{N}^{\prime}(s)=\sum_{k=0}^{N-1}{\hat{f^{\prime}}}_{k} \exp \left(\frac{2 \pi i k x}{L}\right)=\sum_{k=0}^{N-1} i \omega \hat{f}_{k} \exp (i \omega s)$

where $\omega=2 \pi k \Delta x / L=2 \pi k / N$ is the scaled wave number and $s=x / \Delta x$ is the scaled coordinate [11]. For the derivative obtained by a linear scheme, the modified wave number is defined as $\omega^{\prime}=-i\left(\hat{f}^{\prime}{ }_{k}\right) / \hat{f}_{k}$ which can be explicitly obtained by substituting $f_{n}$ with $\hat{f} \exp (i \omega n)$ and $f^{\prime}$ with $i\left(\omega^{\prime}\right) \hat{f} \exp (i \omega n)$ in $f^{\prime}=\frac{1}{\Delta x} \sum_{n} f_{n}$. However, this is not the case for non-linear schemes such as the WENO scheme and the CRWENO scheme. To analyze the spectral properties of non-linear schemes, one should extend the standard Fourier method by considering all Fourier modes rather than only one mode $[35,36]$. The modified wave number $\omega^{\prime}(\omega)$ of a nonlinear scheme is given by:

$\omega^{\prime}(\omega)=\frac{1}{i} \sum_{n=0}^{N-1} f_{n}^{\prime} \exp (-i \omega n) / \sum_{n=0}^{N-1} f_{n} \exp (-i \omega n)$

where $f_{i}^{\prime}$ is the first derivative obtained by a non-linear scheme.

Figs. 2 and 3 show the real and imaginary parts of the modified wave numbers of the WENO-Z scheme, the CRWENO scheme and

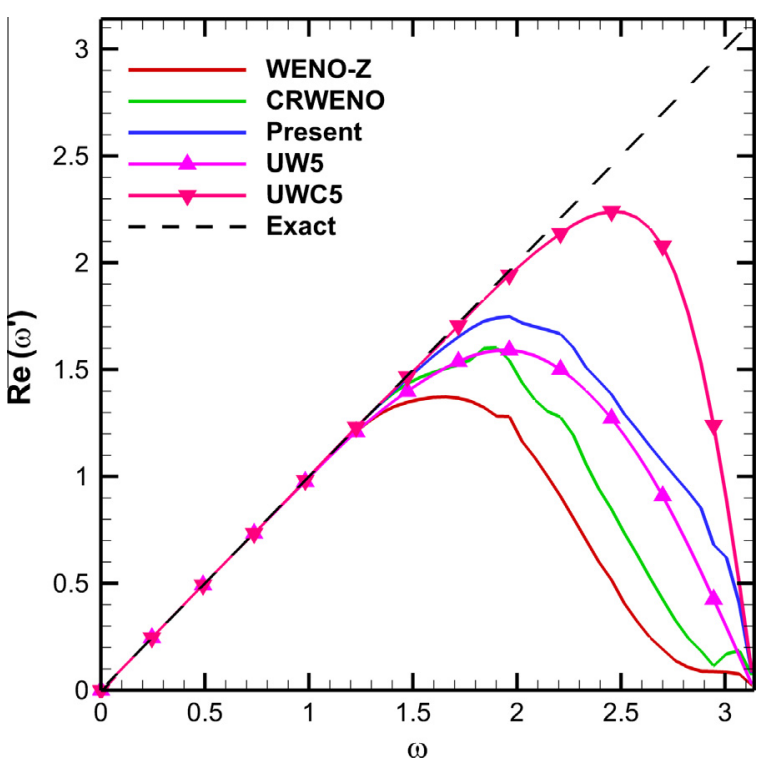

Fig. 2. Real part of the modified wave number for different schemes.

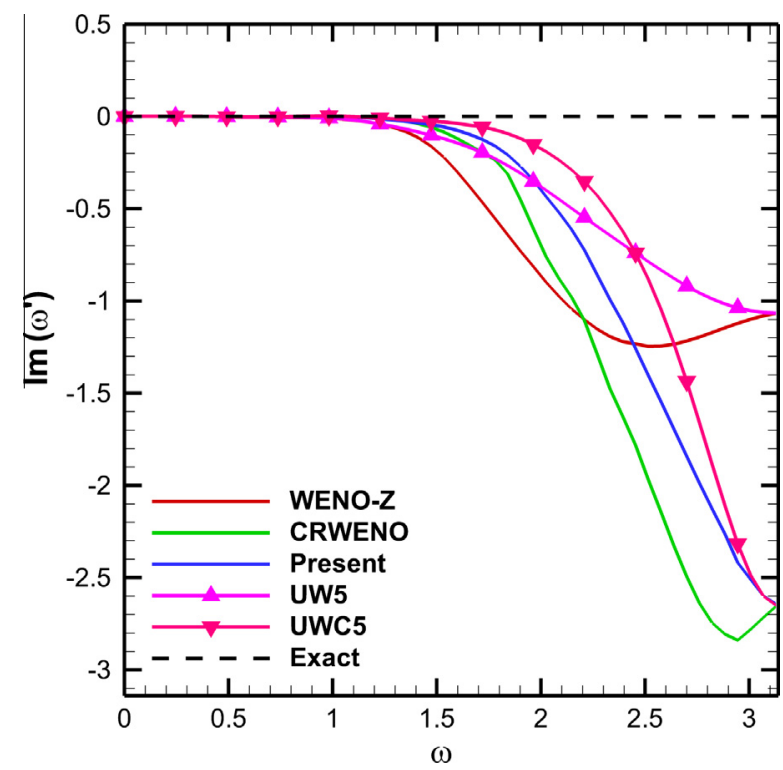

Fig. 3. Imaginary part of the modified wave number for different schemes.

the present method as well as their linear counterparts, i.e., the fifth order upwind scheme (UW5) and the fifth order upwind compact scheme (UWC5). It can be observed that due to the non-linear effects introduced by the weighting functions, the non-linear schemes are more dissipative than their linear counterparts. This observation has also been reported in [37]. The spectral resolution of the CRWENO scheme is effectively improved by the present method.

\section{Numerical experiment}

To assess the present multi-step CRWENO scheme, we perform several numerical tests including scalar, one-dimensional and twodimensional problems. Numerical results are compared with the original CRWENO scheme and the WENO-Z scheme.

For all numerical tests in this section, the third order TVD Runge-Kutta method [38] is used for time advancing:

$u^{(1)}=u^{n}+\Delta t L\left(u^{n}\right)$ 
$u^{(2)}=\frac{3}{4} u^{n}++\frac{1}{4} u^{(1)}+\frac{1}{4} \Delta t L\left(u^{(1)}\right)$

$u^{n+1}=\frac{1}{3} u^{n}+\frac{2}{3} u^{(2)}+\frac{2}{3} \Delta t L\left(u^{(2)}\right)$

For one dimensional problems with strong discontinuities, the characteristic-wise reconstruction is chosen as advised in [31] together with Lax-Friedrich flux splitting [39]. For two dimensional problems, the Steger-Warming flux splitting method [40] is applied.

\subsection{Linear advection equation}

Let us consider the linear wave advection problem. The linear advection equation is given by:

$$
\begin{cases}u_{t}+u_{x}=0 & -1 \leqslant x \leqslant 1 \\ u(x, 0)=u_{0}(x) & \text { periodic boundary }\end{cases}
$$

The exact solution of (43) at time $t$ with the initial condition $u_{0}(x)$ is given by

$u(x, t)=u_{0}(x-t)$

Two cases are studied in this section. The first case is to evaluate the convergence order of the present scheme for a smooth solution. The initial condition is given by:

$u_{0}(x)=\sin \pi x$

A uniformly spaced grid is used as the computational domain. To rule out the error introduced by time discretization, the time step $\Delta t$ is set to $\Delta x^{5 / 3}$.

The $L_{2}$ norm of the error is obtained by comparison with the exact solution at $t=2$ according to:

Table 1

$L_{2}$ errors and convergence orders for different schemes for the linear advection

\begin{tabular}{|c|c|c|c|c|c|c|}
\hline \multirow[t]{2}{*}{$N$} & \multicolumn{2}{|l|}{ WENO-Z } & \multicolumn{2}{|l|}{ CRWENO } & \multicolumn{2}{|l|}{ Present } \\
\hline & $L_{2}$ & Order & $L_{2}$ & Order & $L_{2}$ & Order \\
\hline 20 & $2.26 \mathrm{E}-004$ & - & $8.29 \mathrm{E}-005$ & - & $8.36 \mathrm{E}-005$ & - \\
\hline 40 & $7.08 \mathrm{E}-006$ & 5.00 & $3.31 \mathrm{E}-006$ & 4.78 & $3.31 \mathrm{E}-006$ & 4.78 \\
\hline 80 & $2.22 \mathrm{E}-007$ & 5.00 & $1.10 \mathrm{E}-007$ & 5.01 & $1.10 \mathrm{E}-007$ & 5.01 \\
\hline 160 & $6.98 \mathrm{E}-009$ & 4.92 & $3.20 \mathrm{E}-009$ & 5.85 & $3.20 \mathrm{E}-009$ & 5.85 \\
\hline 320 & $2.41 \mathrm{E}-010$ & 4.86 & $3.29 \mathrm{E}-011$ & 6.60 & $3.29 \mathrm{E}-011$ & 6.60 \\
\hline
\end{tabular}
equation with initial condition (45) at $t=2$.

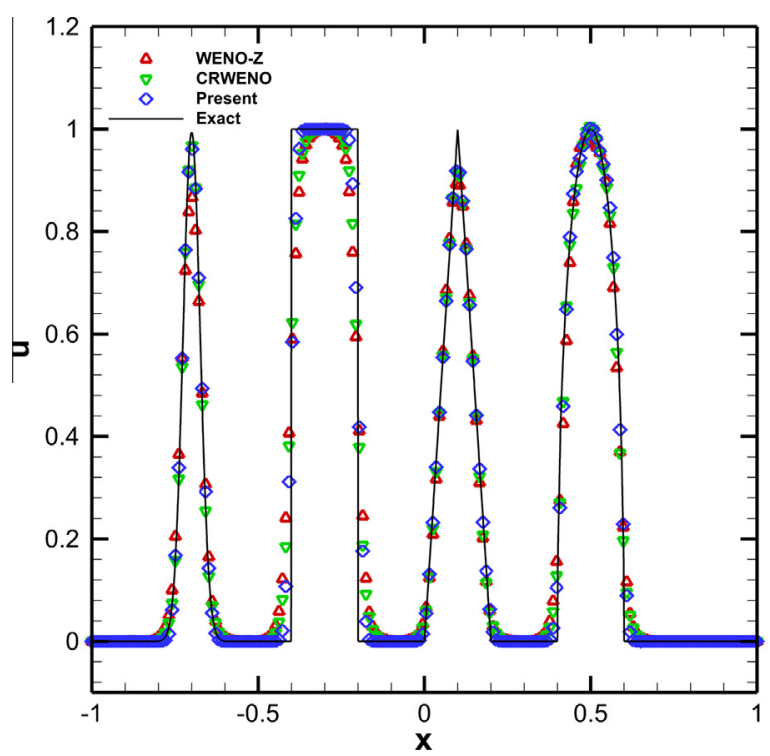

Fig. 4. Solutions of the linear advection equation for different schemes with initial condition (46) at $t=6, N=200$.
$L_{2}=\sqrt{\frac{1}{N} \sum_{i=1}^{N}\left(u_{i}-u_{\text {exact }, i}\right)^{2}}$

Table 1 shows the $L_{2}$ norms as well as convergence orders for different schemes. All schemes achieve fifth order, but the two weighted compact schemes are more accurate than the WENO-Z scheme. Similar accuracy orders are obtained by the CRWENO scheme and the present scheme indicating that the multi-step weighting process designed for shock capturing does not introduce additional error for smooth solution.

The initial condition of the second case is:

$u_{0}(x)= \begin{cases}\frac{1}{6}(G(x, \beta, z-\delta)+G(x, \beta, z+\delta)+4 G(x, \beta, z)), & -0.8 \leqslant x<-0.6 \\ 1, & -0.4 \leqslant x<-0.2 \\ 1-|10(x-0.1)|, & 0 \leqslant x<0.2 \\ \frac{1}{6}(F(x, \alpha, a-\delta)+F(x, \alpha, a+\delta)+4 F(x, \alpha, a)), & 0.4 \leqslant x<0.6 \\ 0, & \text { otherwise }\end{cases}$

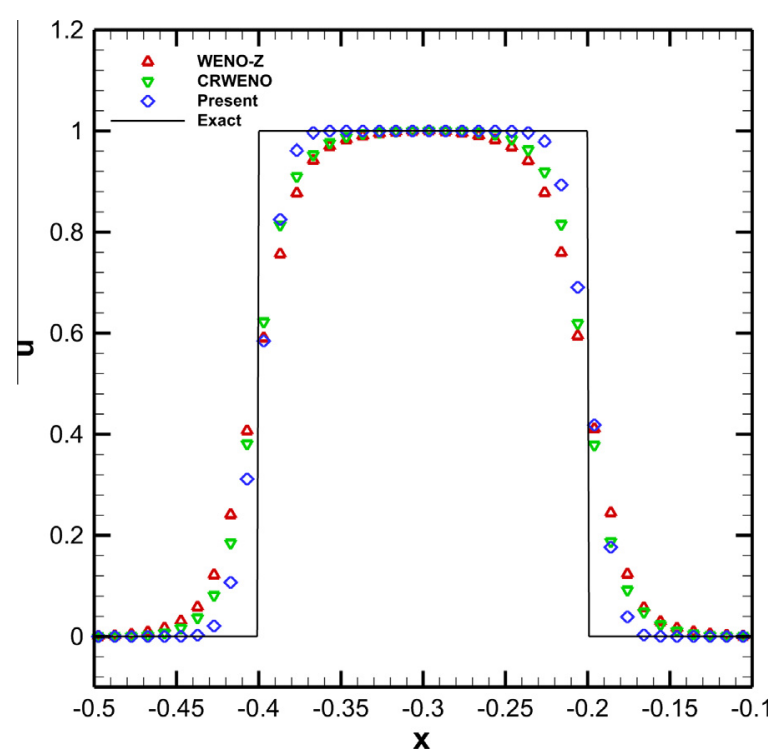

Fig. 5. Detailed plot of the square wave.

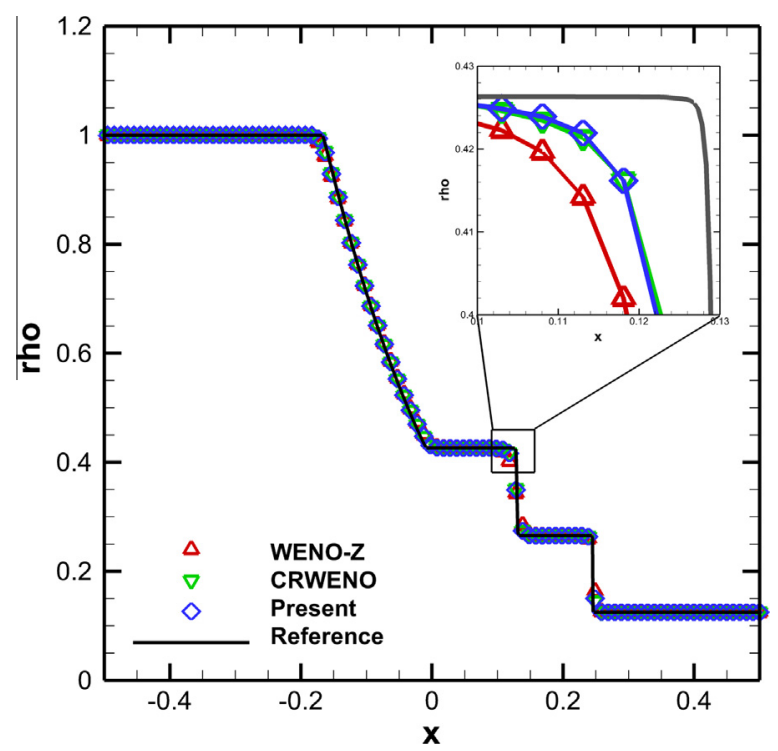

Fig. 6. The Sod problem at $t=0.14, N=200$. 
where

$$
\begin{aligned}
& G(x, \beta, z)=e^{-\beta(x-z)^{2}}, F(x, \alpha, a)=\sqrt{\max \left(1-\alpha^{2}(x-a)^{2}, 0\right)} \\
& a=0.5, z=-0.7, \delta=0.005, \alpha=10, \beta=\log 2 / 36 \delta^{2}
\end{aligned}
$$

The solution of (46) contains a smooth but narrow combination of Gaussians, a square wave, a sharp triangle wave, and a half ellipse [1]. As this test case is a combination of both smooth and nonsmooth functions, it has been widely used to test the discontinuity capturing capability of a scheme [28,41]. Numerical results of different schemes are given in Fig. 4. The present scheme gives an obvious better result at discontinuous points than the other two (see Fig. 5).

\section{2. $1 D$ Euler equations}

The one-dimensional Euler equations are given by $U_{t}+F(U)_{x}=0$

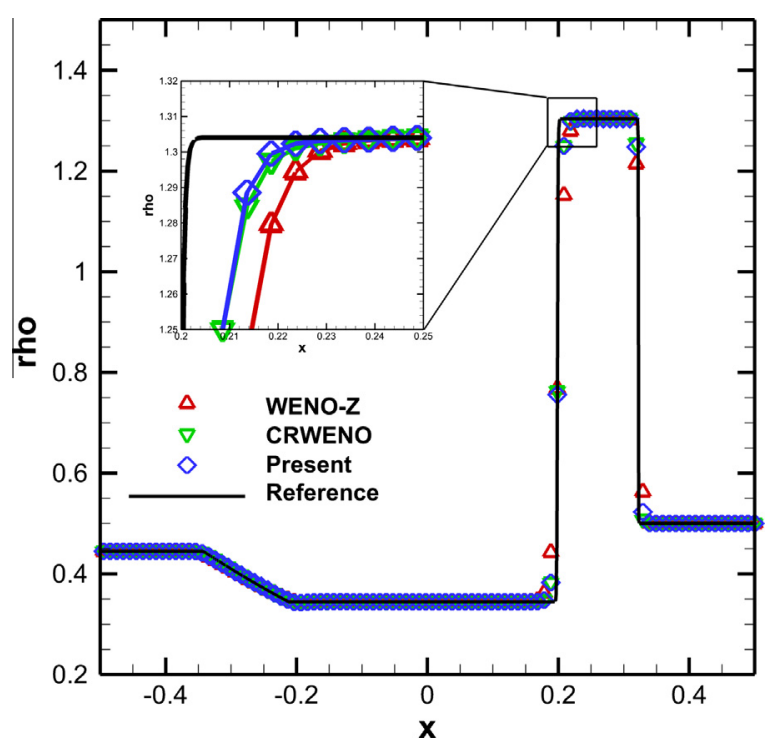

Fig. 7. The Lax problem at $t=0.13, N=200$.

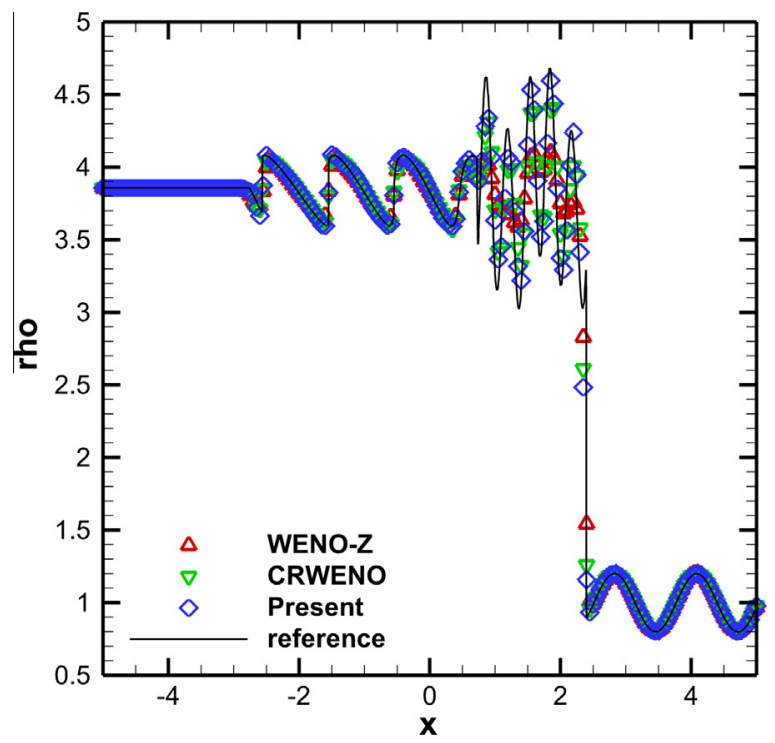

Fig. 8. The Shu-Osher problem at $t=1.8, N=200$. where $U=(\rho, \rho u, e)^{T}, F(U)=\left(\rho u, \rho u^{2}+p, u(e+p)\right)^{T}$. Here $\rho$ is the density, $u$ is the velocity, $e$ is the total energy, $p$ is the pressure, and for ideal gas $e=\frac{p}{\gamma-1}+\frac{1}{2} \rho u^{2}, \gamma=1.4$ is the ratio of specific heat.

Three typical examples are considered. The first example is the Sod problem. The initial condition is:

$(\rho, u, p)= \begin{cases}(1,0,1) & x \leqslant 0 \\ (0.125,0,0.1) & x>0\end{cases}$

with zero gradient boundary conditions at $x= \pm 0.5$.

The second problem is the Lax problem, the initial condition is given by:

$(\rho, u, p)= \begin{cases}(0.445,0.698,3.528) & x \leqslant 0 \\ (0.5,0,0.571) & x>0\end{cases}$

with zero gradient boundary conditions at $x= \pm 0.5$.

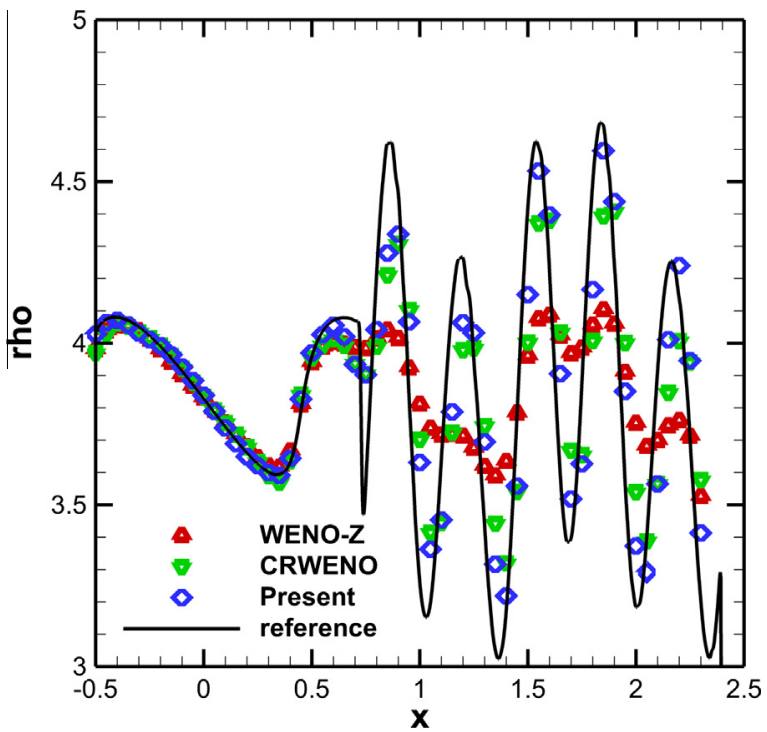

Fig. 9. Detailed plot of the Shu-Osher problem.

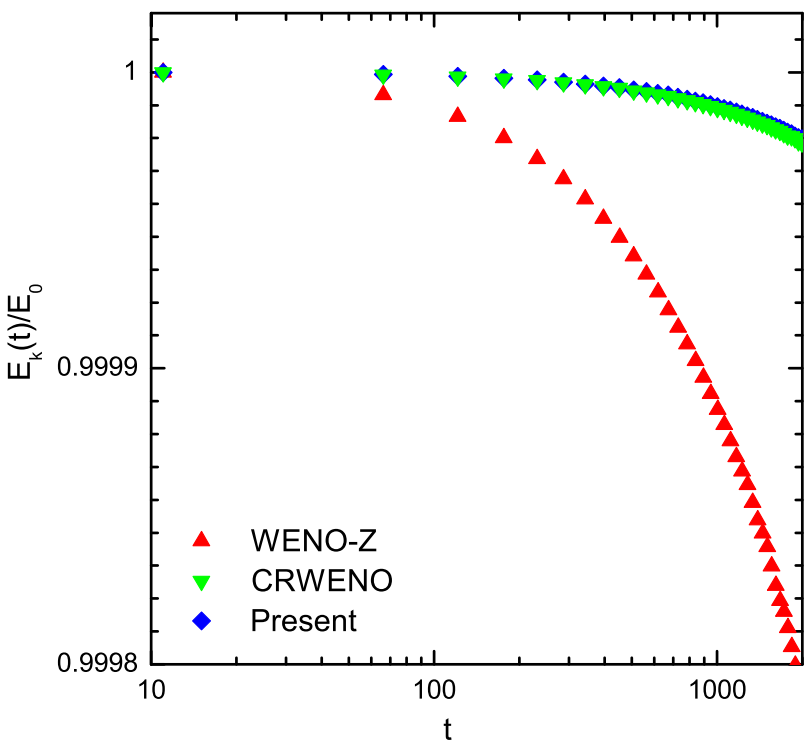

Fig. 10. Integrated kinetic energy versus time for the vortex convection problem. 


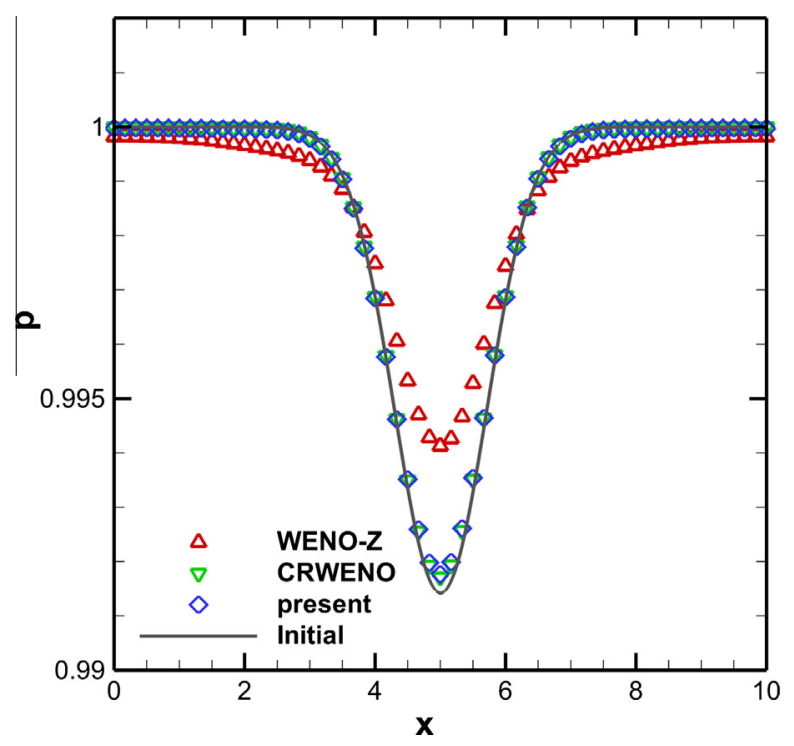

Fig. 11. Pressure distributions at $y=5$ of different schemes at $t=2000$.

The third problem is the Shu-Osher problem. It describes the interaction of a Mach 3 shock with a density wave. The initial condition is given by:

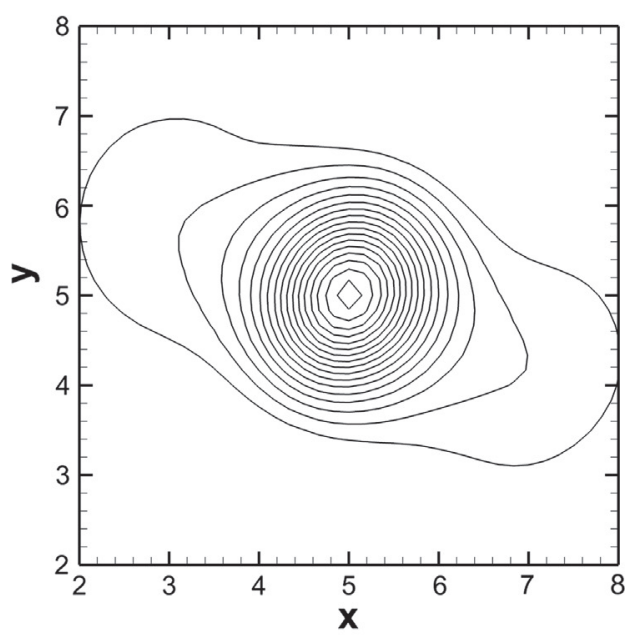

(a) WENO-Z

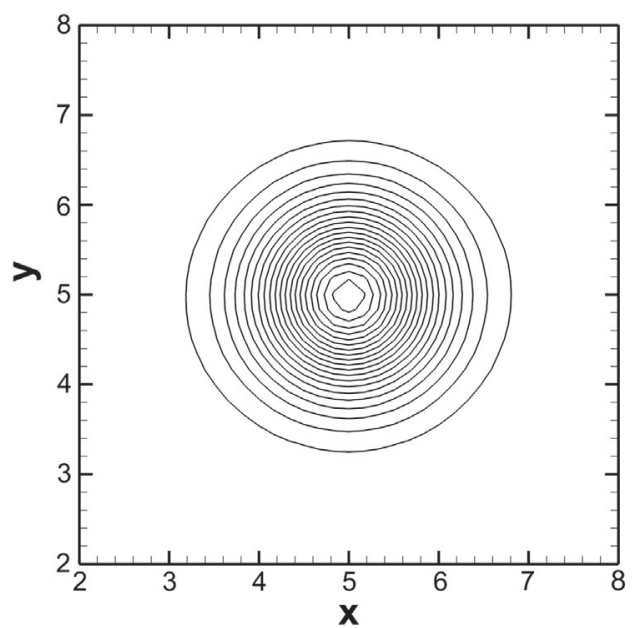

(c) Present

$$
(\rho, u, p)= \begin{cases}\left(\frac{27}{7}, \frac{4 \sqrt{35}}{9}, \frac{31}{3}\right) & x<-4 \\ \left(1+\frac{1}{5} \sin 5 x, 0,1\right) & x \geqslant-4\end{cases}
$$

Zero gradient boundary conditions are applied at $x= \pm 5$.

Figs. 6-9 illustrate the density distributions of the numerical results corresponding to the problems described. The reference result is given by the CRWENO scheme with $N=2000$. For the two shock tube problems, i.e., the Lax problem and the Sod problem, all schemes give similar results. The present scheme and the CRWENO scheme are better than the WENO-Z scheme near shocks. Due to the simple structures of the shocks, the difference between the present scheme and the CRWENO scheme is negligible, although the present method is better than the latter. A more complex problem, the Shu-Osher problem, reveals the difference between tested schemes. The multi-step method gives the best resolution of short waves. Near discontinuities the present method also shows higher accuracy.

\section{3. $2 D$ Euler equations}

The two dimensional Euler equations for ideal gas are given by:

$$
U_{t}+F_{x}+G_{y}=0
$$

where $U, F, G$ vectors are written as:

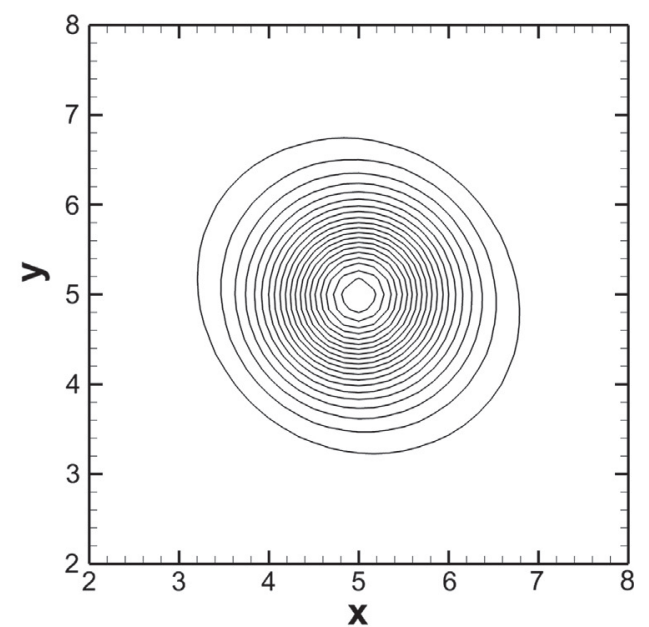

(b) CRWENO

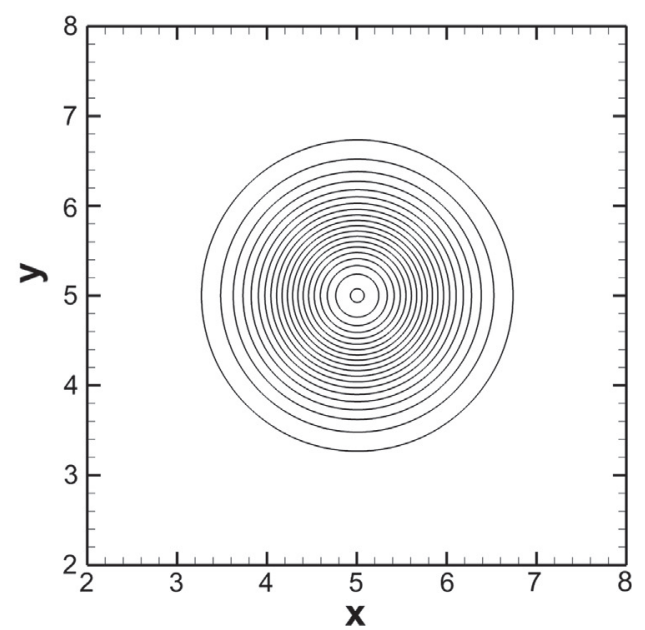

(d) Initial

Fig. 12. Density contours of the isentropic vortex convection problem at $t=2000$ : ranging from 0.9939 to 1 with 20 equally separated levels. 


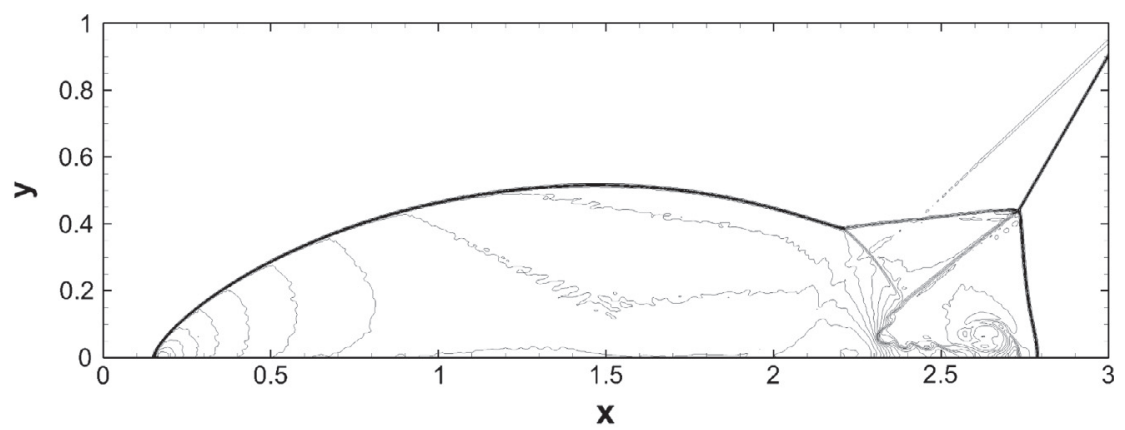

(a) WENO-Z

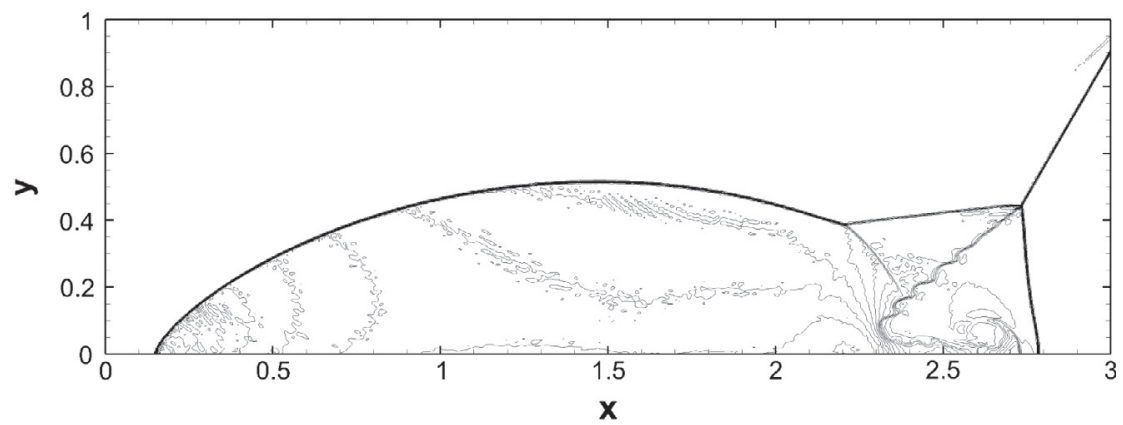

(b) CRWENO

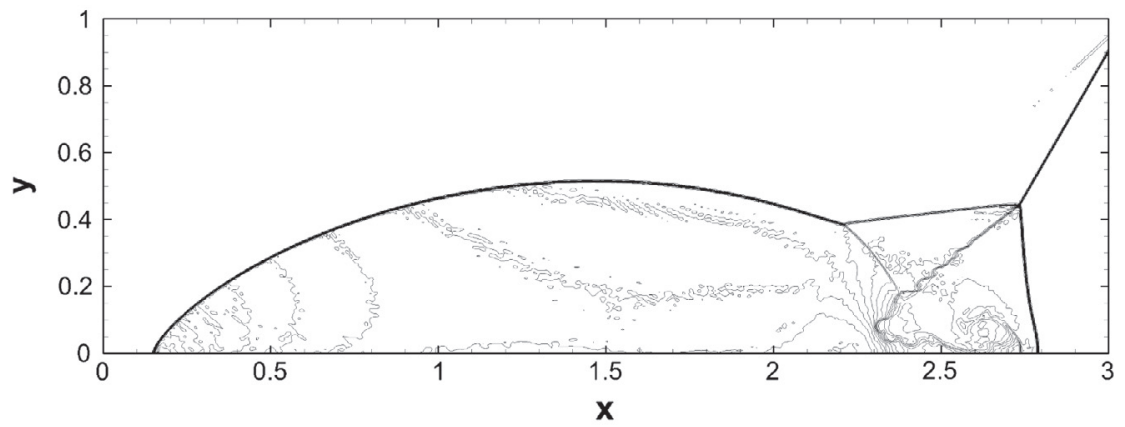

(c) Present

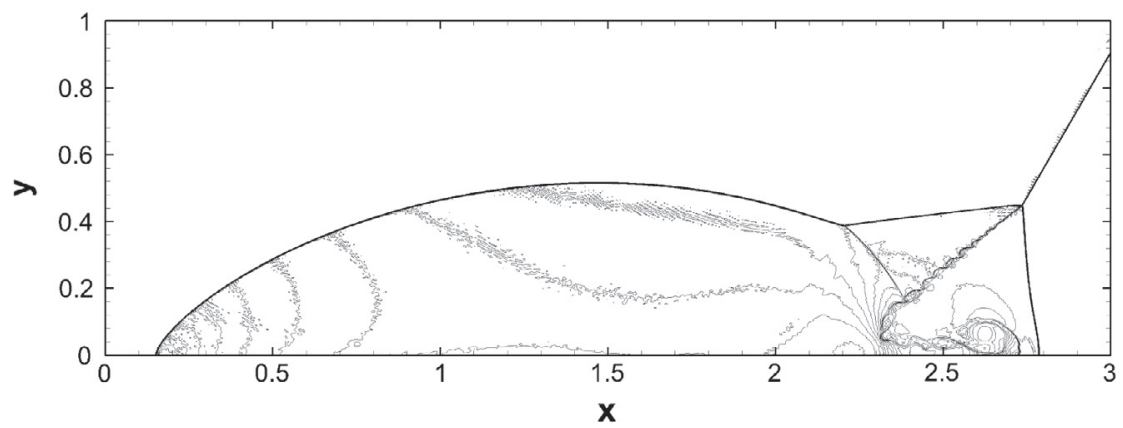

(d) Reference

Fig. 13. Density contours of the double Mach reflection problem at $t=0.2$ : ranging from 20.92 to 1.731 with 30 equally separated levels.

$U=\left[\begin{array}{c}\rho \\ \rho u \\ \rho v \\ e\end{array}\right], \quad F=\left[\begin{array}{c}\rho u \\ \rho u^{2}+p \\ \rho u v \\ e u+p u\end{array}\right], \quad G=\left[\begin{array}{c}\rho v \\ \rho u v \\ \rho v^{2}+p \\ e v+p v\end{array}\right]$
In this section two problems are tested with WENO-Z, CRWENO and the present multi-step method. The first problem is to simulate an isentropic vortex moving across the computational domain periodically. This test was given in [42] and used to test the dissipation of a scheme for a smooth solution of the Euler equations. The second test is the double Mach reflection problem. Except for 
strong shocks, the solution of the double Mach reflection problem also contains small structures. Thus it is a good case to test the small scale resolution of a scheme with discontinuities.

\subsubsection{Isentropic vortex convection}

This problem is used to test the dissipation of a scheme [42]. An isentropic vortex moves across the computational domain periodically. The computational domain is $[0,10] \times[0,10]$ with the free stream specified as $\rho_{\infty}=1, u_{\infty}=0.5, v_{\infty}=0, p_{\infty}=1$. The initial flow condition is given by:

$$
\begin{aligned}
& \rho=\left[1-\frac{(\gamma-1) b^{2}}{8 \gamma \pi^{2}} e^{1-r^{2}}\right]^{\frac{1}{\gamma-1}} ; p=\rho^{\gamma} \\
& \delta u=-\frac{b}{2 \pi} e^{\frac{1-r^{2}}{2}}\left(y-y_{c}\right) \\
& \delta v=\frac{b}{2 \pi} e^{\frac{1-r^{2}}{2}}\left(x-x_{c}\right)
\end{aligned}
$$

where $r^{2}=\left(x-x_{c}\right)^{2}+\left(y-y_{c}\right)^{2}$ is the distance from the vortex center $\left(x_{c}, y_{c}\right)=(5,5)$ and the vortex strength $b$ is 0.5 . Periodic boundary conditions are applied to all boundaries. Dissipation is measured by the integrated kinetic energy as time evolves: $E_{k}=\sum_{i, j} 0.5 \rho_{i, j}\left(u_{i, j}^{2}+v_{i, j}^{2}\right)$.

Fig. 10 shows the integrated kinetic energy evolution of different schemes, the multi-step method and the CRWENO scheme are significantly less dissipative than the WENO-Z scheme. This result can also be observed in Fig. 11, the pressure value at the vortex center of the WENO-Z scheme deviates largely from the initial value. The minimum pressure of the multi-step method and CRWENO is almost the same. Therefore, with respect to dissipation, as shown in $1 \mathrm{D}$ linear advection case, the multi-step method does not introduce additional error for two-dimensional smooth solution.

However, the vortex shape maintaining capability is important for long time simulation such as direct numerical simulation and large eddy simulation. From the density contours for different schemes at $t=2000$ shown in Fig. 12, it can be seen that the vortex

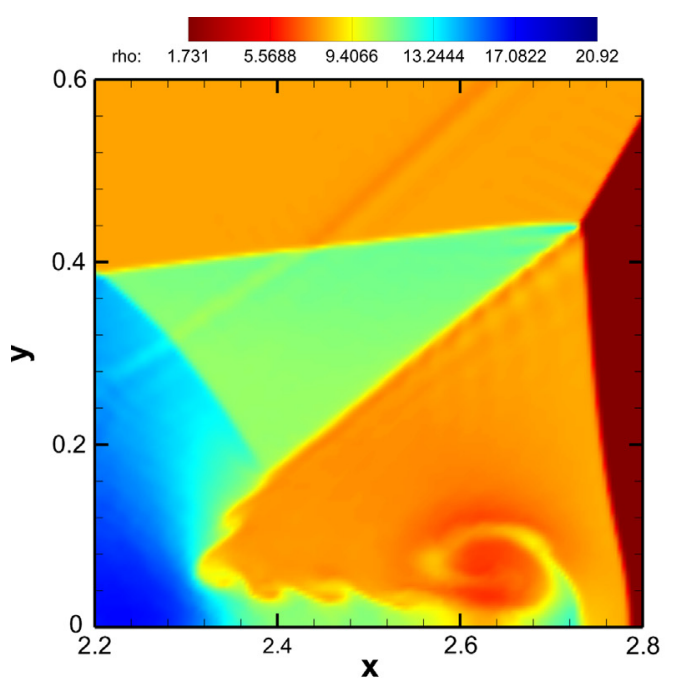

(a) WENO-Z

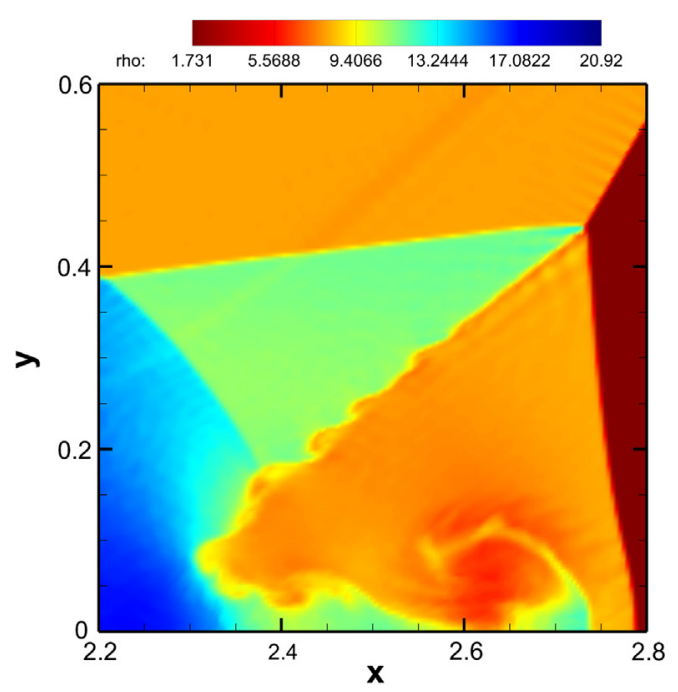

(c) Present

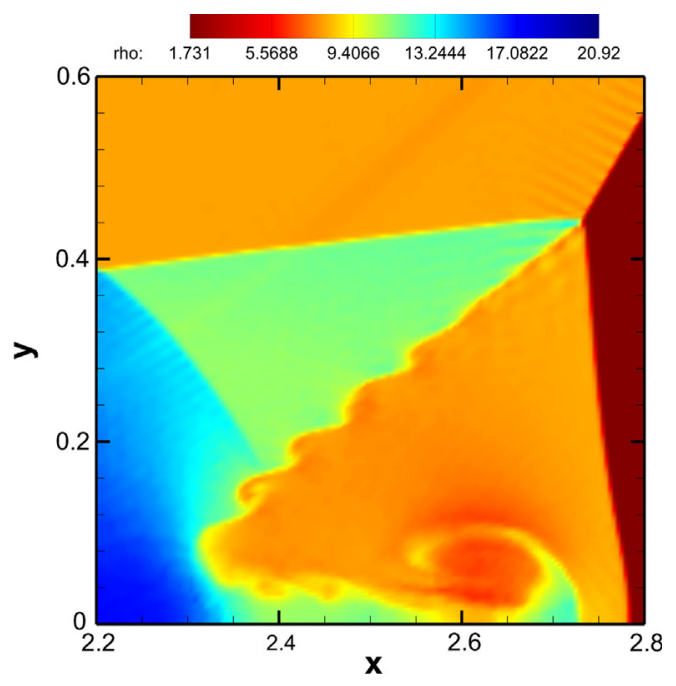

(b) CRWENO

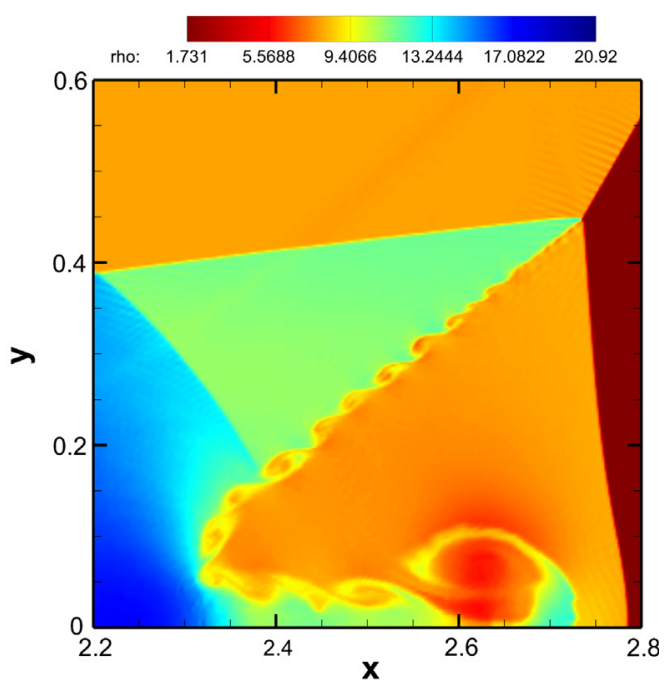

(d) Reference

Fig. 14. Density contours of the double Mach reflection problem in the up-rolling region at $t=0.2$. 
shape of the WENO-Z scheme is substantially distorted and the CRWENO scheme gives a slightly distorted distribution. The multi-step method gives the CRWENO scheme even better shape maintaining capability. As the CRWENO scheme and the present scheme give the same dissipation rate, a better vortex shape maintaining capability indicates that the present multi-step method is more suitable for long time complex flow simulation.

\subsubsection{Double Mach reflection}

The double Mach reflection test is a mimic of the planar shock reflection in the air from wedges. It is a widely used benchmark to test the ability of shock capturing and the small scale structure resolution of a certain scheme. In the present simulation, the computational domain is $[0,4] \times[0,1]$. The lower boundary is set to be reflecting wall starting from $x=\frac{1}{6}$. At $t=0$, a right-moving $60^{\circ}$ inclined Mach 10 shock is positioned at $\left(\frac{1}{6}, 0\right)$. The upper boundary is set to describe the exact motion of the Mach 10 shock. The left boundary at $x=0$ is assigned with post-shock values. An outflow condition with zero gradients is set at $x=4$. Readers may refer to [43] for detailed description of the double Mach reflection problem. An uniform grid is used with $\Delta x=\Delta y=\frac{1}{240}$. The reference result is given by the CRWENO scheme with $\Delta x=\Delta y=\frac{1}{480}$.

Fig. 13 shows the density contours at $t=0.2$ obtained with different schemes. The shock waves are well captured by each scheme. Zonal magnifications of the up-rolling region of each result are shown in Fig. 14. Compared with the reference result given by the CRWENO scheme, the present multi-step scheme shows better resolution. The WENO-Z scheme is able to capture a few small structures near the up-rolling region, but does not resolve these structures along the contact line due to its low

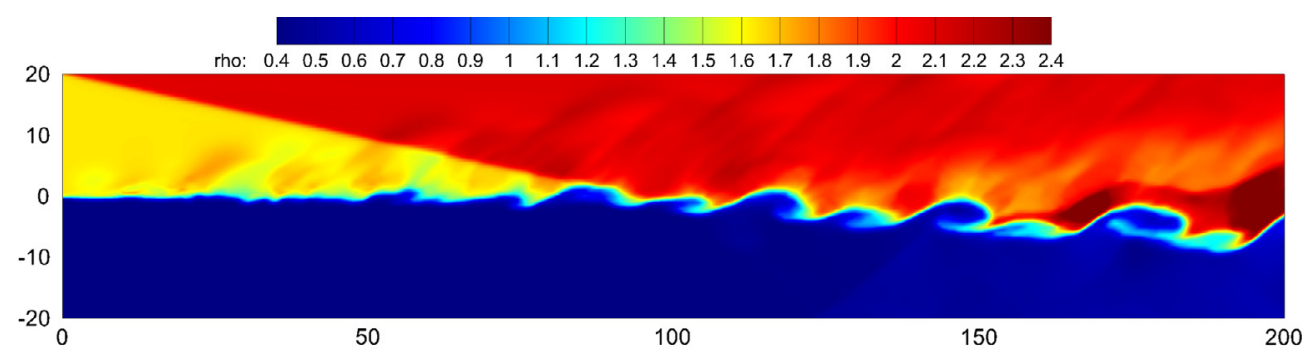

(a) WENO-Z

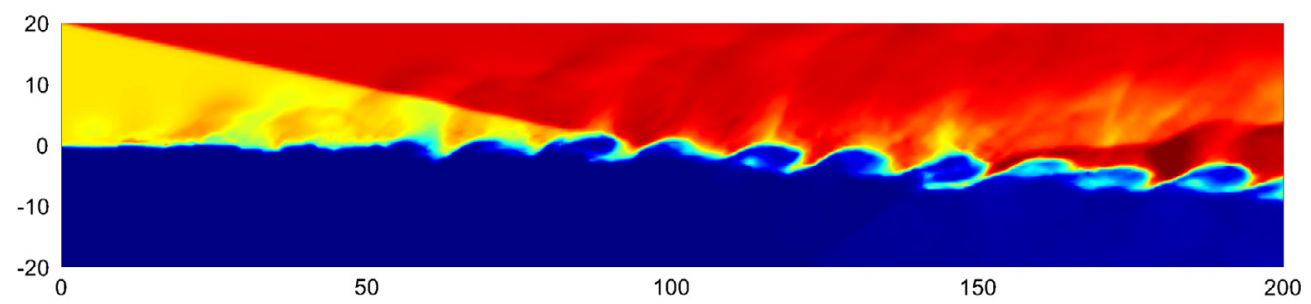

(b) CRWENO

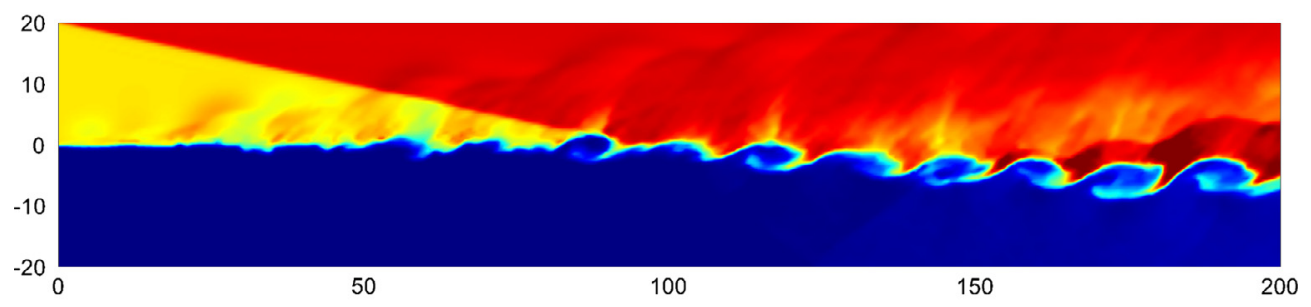

(c) Present

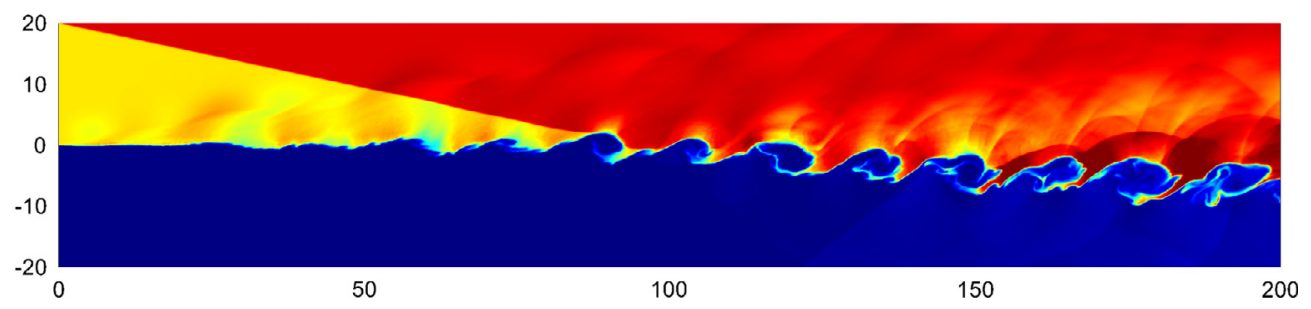

(d) Reference

Fig. 15. Density contours of the shock/mixing layer interaction problem at $t=120$. 
accuracy near shock. The CRWENO scheme also shows an unsatisfactory accuracy near discontinuities as analyzed in Section 3 but still captures some vortex-like structures along the contact line. The present method resolves more small structures along the contact line. This implies that the multi-step method is more accurate and less dissipative near discontinuities.

\subsection{Shock wave impingement on a spatially evolving mixing layer}

The shock wave impingement problem is designed to measure the resolution of schemes when shock waves interact with vortices [44]. A Mach 0.6 mixing layer evolves and impacts on an oblique shock. The vortices produced by the mixing layer instability pass, firstly, through the oblique shock and then a second shock reflected by the slip wall at the lower boundary. The computational domain is $[0,200] \times[-20,20]$. At $x=0$, the inlet condition is specified as:

$u=2.5+0.5 \tanh (2 y)$

For the upper stream $(y>0), \rho=1.6374, p=0.3327$ and for the lower stream $(y<0), \rho=0.3626, p=0.3327$. The post shock condition is set at the upper boundary, and the slip wall condition is applied at the lower boundary. Fluctuations are added to the vertical velocity component at the inlet:

$v^{\prime}=\sum_{k=1}^{2} a_{k} \cos \left(2 \pi k t / T+\phi_{k}\right) \exp \left(-y^{2} / b\right)$

$b=10, a_{1}=a_{2}=0.05, \phi_{1}=0, \phi_{2}=\pi / 2$

in which $T=\lambda / u_{c}$ is the period, $\lambda=30$ is the wavelength, $u_{c}=2.68$ is the convective velocity. The Prandtl number $P r$ is set to 0.72 and the Reynolds number Re is chosen to be 500 .

The two dimensional Navier-Stokes equations for ideal gas are given by:

$U_{t}+F_{x}+G_{y}=\frac{1}{R e} F_{x}^{v}+\frac{1}{R e} G_{y}^{v}$

where $U, F, G, F_{v}, G_{v}$ vectors are written as:

$$
\begin{gathered}
U=\left[\begin{array}{c}
\rho \\
\rho u \\
\rho v \\
e
\end{array}\right], F=\left[\begin{array}{c}
\rho u \\
\rho u^{2}+p \\
\rho u v \\
e u+p u
\end{array}\right], G=\left[\begin{array}{c}
\rho v \\
\rho u v \\
\rho v^{2}+p \\
e v+p v
\end{array}\right], \\
F^{v}=\left[\begin{array}{c}
0 \\
\tau_{x x} \\
\tau_{x y} \\
u \tau_{x x}+v \tau_{x y}+q_{x}
\end{array}\right], G^{v}=\left[\begin{array}{c}
\tau_{y y} \\
u \tau_{y x}+v \tau_{y y}+q_{y}
\end{array}\right]
\end{gathered}
$$

in which

$$
\begin{aligned}
& \tau_{x x}=\mu\left(\frac{4}{3} \frac{\partial u}{\partial x}-\frac{2}{3} \frac{\partial v}{\partial y}\right), \quad \tau_{y y}=\mu\left(\frac{4}{3} \frac{\partial v}{\partial y}-\frac{2}{3} \frac{\partial u}{\partial x}\right) \\
& \tau_{x y}=\tau_{y x}=\mu\left(\frac{\partial u}{\partial y}+\frac{\partial v}{\partial x}\right) \\
& q_{x}=\mu \frac{1}{(\gamma-1) M^{2} \operatorname{Pr}} \frac{\partial T}{\partial x} \\
& q_{y}=\mu \frac{1}{(\gamma-1) M^{2} \operatorname{Pr}} \frac{\partial T}{\partial y}
\end{aligned}
$$

The viscous terms are discretized with the fourth order central difference scheme [45]. The grid number is $321 \times 81$ and a refined grid of $1284 \times 324$ is used as reference. The reference case is computed with the CRWENO scheme.

Fig. 15 shows the density contours of the numerical results of different schemes. The result of the multi-step CRWENO scheme resembles the reference result most compared to the other two schemes. The vortices near the oblique shock and the reflecting shock are better resolved by the present method. Besides, the shapes of vortices near the outflow boundary are also well maintained by the present multi-step method.

\section{Conclusion and discussion}

In this paper, we employ a multi-step method to improve a recently proposed weighted compact scheme, i.e., the CRWENO scheme. Accuracy analysis of points near discontinuities shows that the accuracy of the CRWENO scheme is second order. By introducing the multi-step method, the accuracy at such circumstance is improved to third order. Numerical tests show that the present multi-step scheme has lower dissipation and higher resolution in both smooth and discontinuous regions. For long time simulation such as direct and large eddy simulation of supersonic turbulent flow, the present method may provide more accurate results.

It is worth noting that, compared to the original scheme, the multi-step CRWENO scheme calculates three more weights. This results in a 50 percent increase in the computation time. However, for an realistic large scale simulation of supersonic turbulence, global multi-step weighting is not necessary. To decrease computational expense, one may employ a switch function such as those used in hybrid schemes which only turns on the multi-step method at points near discontinuities. Different from hybrid schemes, as multi-step and one step weighting schemes are essentially the same, switching between these two schemes does not lead to lowered accuracy and poor resolution. This switch function is fairly easy to realize that only a few additional lines are needed for an existing multi-step scheme code.

Finally, the multi-step strategy is an efficient method to improve the accuracy of the CRWENO scheme. It can also be applied to other weighted compact schemes which have the same deficiency as the CRWENO scheme. Application of the multi-step CRWENO scheme in supersonic turbulence simulation will be presented in future works.

\section{Acknowledgement}

This research work was supported by the National Natural Science Foundation of China under Grant 11272325.

\section{References}

[1] Jiang G-S, Shu C-W. Efficient implementation of weighted ENO schemes. J Comput Phys 1996;126(1):202-28.

[2] Harten A, Engquist B, Osher S, Chakravarthy SR. Uniformly high order accurate essentially non-oscillatory schemes, III. J Comput Phys 1987;71(2):231-303.

[3] Liu X-D, Osher S, Chan T. Weighted essentially non-oscillatory schemes. J Comput Phys 1994;115(1):200-12.

[4] Harten A. High resolution schemes for hyperbolic conservation laws. J Comput Phys 1983;49(3):357-93.

[5] Johnsen E, Larsson J, Bhagatwala AV, Cabot WH, Moin P, Olson BJ, et al. Assessment of high-resolution methods for numerical simulations of compressible turbulence with shock waves. J Comput Phys 2010;229(4):1213-37.

[6] Henrick AK, Aslam TA, Powers JM. Mapped weighted essentially nonoscillatory schemes: achieving optimal order near critical points. J Comput Phys 2005;207(2):542-67.

[7] Borges R, Carmona M, Costa B, Don WS. An improved weighted essentially non-oscillatory scheme for hyperbolic conservation laws. J Comput Phys 2008;227(6):3191-211.

[8] Yamaleev NK, Carpenter MH. A systematic methodology for constructing highorder energy stable WENO schemes. J Comput Phys 2009;228(11):4248-72.

[9] Martin MP, Taylor EM, Wu M, Weirs VG. A bandwidth-optimized WENO scheme for the effective direct numerical simulation of compressible turbulence. J Comput Phys 2006;220(1):270-89.

[10] Shen Y, Zha G. Improvement of weighted essentially non-oscillatory schemes near discontinuities. Comput Fluids 2014;96(0):1-9.

[11] Lele SK. Compact finite difference schemes with spectral-like resolution. J Comput Phys 1992;103(1):16-42. 
[12] Gamet L, Ducros F, Nicoud F, Poinsot T, et al. Compact finite difference schemes on non-uniform meshes. Application to direct numerical simulations of compressible flows. Int J Numer Methods Fluids 1999;29(2):159-91.

[13] Samtaney R, Pullin DI, Kosovic B. Direct numerical simulation of decaying compressible turbulence and shocklet statistics. Phys Fluids 2001; 13(5):1415-30.

[14] Constantinescu GS, Lele SK. Large eddy simulation of a near sonic turbulent jet and its radiated noise. AIAA Paper 2001;376:2001.

[15] Levy D, Puppo G, Russo G. Compact central WENO schemes for multidimensional conservation laws. SIAM J Sci Comput 2000;22(2):656-72.

[16] Kim JK. Optimised boundary compact finite difference schemes for computational aeroacoustics. J Comput Phys 2007;225(1):995-1019.

[17] Tam CKW, Webb JC. Dispersion-relation-preserving finite difference schemes for computational acoustics. J Comput Phys 1993;107(2):262-81.

[18] Fu D, Ma Y. A high order accurate difference scheme for complex flow fields. Comput Phys 1997;134(1):1-15.

[19] Deng X, Maekawa H. Compact high-order accurate nonlinear schemes. Comput Phys 1997;130(1):77-91.

[20] Tolstykh AI, Lipavskii MV. On performance of methods with third-and fifthorder compact upwind differencing. J Comput Phys 1998;140(2):205-32.

[21] Shah A, Yuan L, Khan A. Upwind compact finite difference scheme for timeaccurate solution of the incompressible Navier-Stokes equations. Appl Math Comput 2010;215(9):3201-13.

[22] Adams NA, Shariff K. A high-resolution hybrid compact-ENO scheme fo shock-turbulence interaction problems. J Comput Phys 1996;127(1):27-51.

[23] Pirozzoli S. Conservative hybrid compact-WENO schemes for shockturbulence interaction. J Comput Phys 2002;178(1):81-117.

[24] Ren Y-X, Liu M, Zhang H. A characteristic-wise hybrid compact-WENO scheme for solving hyperbolic conservation laws. J Comput Phys 2003:192(2):365-86.

[25] Hill DJ, Pullin DI. Hybrid tuned center-difference-WENO method for large eddy simulations in the presence of strong shocks. J Comput Phys 2004: 194(2):435-50.

[26] Kim D, Kwon JH. A high-order accurate hybrid scheme using a central flux scheme and a WENO scheme for compressible flowfield analysis. J Comput Phys 2005;210(2):554-83.

[27] Zhang S, Jiang S, Shu C-W. Development of nonlinear weighted compact schemes with increasingly higher order accuracy. J Comput Phys 2008; 227(15):7294-321.

[28] Shen Y, Zha G. Generalized finite compact difference scheme for shock/complex flowfield interaction. J Comput Phys 2011:230(12):4419-36.

[29] Jiang L, Shan H, Liu C. Weighted compact scheme for shock capturing. Int J Comput Fluid Dyn 2001;15(2):147-55.
[30] Deng X, Zhang H. Developing high-order weighted compact nonlinear schemes. J Comput Phys 2000;165(1):22-44.

[31] Ghosh D, Baeder JD. Compact reconstruction schemes with weighted ENO limiting for hyperbolic conservation laws. SIAM J Sci Comput 2012; 34(3):A1678-706.

[32] Ghosh D, Baeder JD. Weighted non-linear compact schemes for the direct numerical simulation of compressible, turbulent flows. J Sci Comput 2014:1-29.

[33] Lee S, Lele SK, Moin P. Eddy shocklets in decaying compressible turbulence. Phys Fluids 1991;3(4):657-64.

[34] Lee S, Lele SK, Moin P. Interaction of isotropic turbulence with shock waves: effect of shock strength. J Fluid Mech 1997;340:225-47.

[35] Zhao S, Lardjane N, Fedioun I. Comparison of improved finite-difference WENO schemes for the implicit large eddy simulation of turbulent non-reacting and reacting high-speed shear flows. Comput Fluids 2014;95:74-87.

[36] Pirozzoli S. On the spectral properties of shock-capturing schemes. J Comput Phys 2006;219(2):489-97.

[37] Jia F, Gao Z, Don W. A spectral study on the dissipation and dispersion of the WENO schemes. J Sci Comput 2014;63(1):49-77.

[38] Shu C-W. Total-variation-diminishing time discretizations. SIAM J Sci Stat Comput 1988;9(6):1073-84.

[39] Lax PD. Weak solutions of nonlinear hyperbolic equations and their numerical computation. Commun Pure Appl Math 1954:7(1):159-93.

[40] Steger SL, Warming RF. Flux vector splitting of the inviscid gasdynamic equations with application to finite-difference methods. J Comput Phys 1981;40(2):263-93.

[41] Balsara D, Shu C-W. Monotonicity preserving weighted essentially nonoscillatory schemes with increasingly high order of accuracy. J Comput Phys 2000;160(2):405-52.

[42] Davoudzadeh F, McDonald H, Thompson BE. Accuracy evaluation of unsteady CFD numerical schemes by vortex preservation. Comput Fluids 1995; 24(8):883-95.

[43] Woodward P Colella P. The numerical simulation of two-dimensional fluid flow with strong shocks. J Comput Phys 1984;54(1):115-73.

[44] Yee HC, Sandham ND, Djomehri MJ. Low-dissipative high-order shockcapturing methods using characteristic-based filters. J Comput Phys 1999; 150(1):199-238.

[45] Shen Y, Zha G, Chen X. High order conservative differencing for viscous terms and the application to vortex-induced vibration flows. J Comput Phys 2009; 228(22):8283-300. 